\title{
REACH: A Challenge for the Swiss Chemical Industry!
}

\author{
Richard Gamma and Paul Vesel ${ }^{\star}$
}

\begin{abstract}
REACH is currently one of the EU's most far-reaching pieces of proposed legislation. The political goals of this proposal are: the protection of human health and environment, the maintenance of the competitiveness of European Chemical industry and to prevent a break-up of the single market. These political goals are basically supported by the chemical industry. However the regulation is far away from any practicable form of legislation. The proposals are expensive, complicated and too bureaucratic for companies. Small and medium-sized enterprises will hardly be able to follow these demands. The special situation of chemical industry in Switzerland, as a non EU country - will be discussed.
\end{abstract}

Keywords: REACH · Swiss Society of Chemical Industries

\section{Introduction}

Chemical regulations are nothing new, either in the EU and its member states or in Switzerland. However, the legal proposal for the registration, evaluation and authorisation of chemicals (REACH) adds a new dimension to a system of chemical legislation that is already dense and comprehensive in the EU. The political process is well advanced and the REACH regulation is expected to come into force by spring or summer 2007. The question of its potential effects on Switzerland thus arises. Following is a description of how Swiss companies

*Correspondence: Dr. P. Vesel

SGCI Chemie Pharma Schweiz

(Swiss Society of Chemical Industries)

Nordstrasse 15

$\mathrm{CH}-8035$ Zürich

Tel.: +4144368 1734

Fax: +41443681735

E-Mail: paul.vesel@sgci.ch in the chemical and pharmaceutical industry as well as their customers at home and abroad might be affected and which options exist for companies in Switzerland.

SGCI Chemie Pharma Schweiz (Swiss Society of Chemical Industries; www.sgci. $c h$ ) is the umbrella organisation of the chemical and pharmaceutical industry in Switzerland. The 180 member companies are active in research and development, production and sale of all kind of chemical and pharmaceutical products. The Swiss chemical and pharmaceutical industry operates nearly exclusively in the field of specialties. Today $90 \%$ of the Swiss chemical industry's overall product portfolio are specialties; a remarkable portion compared to international average. Producing more than 30000 products, it is exceptionally differentiated. The following major product groups can be distinguished in terms of areas of application: Pharmaceuticals and Diagnostics, Fine chemicals Vitamins, Flavours and Fragrances, Crop protection agents, Specialty chemicals for industrialtechnical purposes, Pigments, Paints and Lacquers.

SGCI Chemie Pharma Schweiz is committed to close collaboration with similar organisations of the chemical and pharmaceutical industries in other countries; it is an active member of a large number of industry associations, in Europe as well as internationally. Experts of SGCI Chemie Pharma Schweiz and their member companies are active in working groups dealing with European and international chemical policy, especially participating in the work of the European Chemical Association CEFIC on political strategy, advocacy and implementation of REACH.

\section{Initial Situation}

\subsection{REACH Goals}

REACH is currently one of the EU's most far-reaching pieces of proposed legislation. On 29th October 2003, the Commission passed its draft regulation for the registration, evaluation and authorisation of chemicals [1] and submitted it to the EU Parliament and the Council of Ministers for further discussion. Among other things, the Commission describes the political goals of its proposal as being the protection of human and environmental health, the maintenance and strengthening of the competitiveness of the European chemical industry and the desire to prevent a breakup of the single market. These political goals are also supported, in principle, by the chemical industry. Product stewardship as a central, product-related element sees the implementation of the idea of RESPONSIBLE CARE, with which the chemical and pharmaceutical industry worldwide contribute to sustainable development. The safe handling of chemical substances throughout their entire lifecycle, i.e. from their manufacture and use to their disposal, is supported by the companies.

\subsection{Current Status of REACH Consultations}

In its decision of 17 th November 2005 , the European Parliament passed a series of proposed amendments to the commission's 
draft ordinance [2]. In particular, the decisions for a more workable and more costefficient registration procedure are welcomed. However, Parliament also adopted a number of highly problematical changes at the same time, in particular, regarding the authorization process, e.g. the limited duration of authorisations (five years) and the obligation to substitute, irrespective of the safe use of substance. The evidence of safe use of a substance is not sufficient for the granting of an authorisation. According to the proposal the applicant must additionally prove that no alternative substances or technologies are available and that socioeconomic benefits outweigh possible risks. According the EP (EP, Article 57(6)) the authorisation is generally limited to five years at most. 18 months before the expiry of this time limit, a new application must be submitted; otherwise authorisation become automatically invalid. This would cause immense legal uncertainty in investment decisions and corporate planning. Moreover, companies and competent authorities would be constantly burdened with applications to extend authorisations granted for limited time periods.

Highly crucial are also the tighter requirements to disclose information, connected with a reduced protection of confidential business information.

The Competitiveness Council reached political agreement on 13th December 2005 [3]. The European chemical industry sees the Council decisions as an improvement over the Commission Proposal, on which these decisions are based. In particular, CEFIC welcomes that the Council largely took back and corrected the extremely problematic tightening of rules for the authorisation procedure, as had been adopted by the EP. At the same time, however, the Council decision brought the EP's relatively balanced registration package back in a more work and cost-intensive form. This largely relates to low-volume special products in the range of $1-100 \mathrm{t} / \mathrm{y}$, i.e. an area in which speciality chemicals, in particular, would be affected in Switzerland, too. For example the dataset according Annex $\mathrm{V}$ was enhanced with an algae-test, compared to the version of EP. For low volume chemicals this test would not yield more really relevant information, which is important for safe use. The screening test for reprotoxicity is again reintroduced. This test is extremely expensive ( $c a .50000$ Euro) and would increase the requirement for laboratory animals.

The Council of Ministers passed the Common Position on 27th June 2006 [4]. There are substantial differences between the European Parliament version and that of the Common Position. The second reading in the European Parliament is scheduled for October 2006.

\subsection{Basic Assessment of the Current Draft by the European Chemical Industry}

The political goals formulated by the commission are basically supported by the chemical industry. However, the regulation together with the attachments is more than 1000 (one thousand!) pages and is far from any practicable form of legislation. The proposals are expensive, complicated and too bureaucratic for companies. Small and medium-sized enterprises, in particular, will hardly be able to follow such demands. This has been correspondingly proven by test projects on the practicability of REACH in different countries, as well as in the entire European Union.

The European Parliament and the Competitiveness Council have definitely improved a number of points of the EU committee proposal that are important where practicability and applicability are concerned. Examples: The scope has changed; wastes were exempted entirely from REACH; minerals, ores, concentrate and several other substances and categories of substance are exempted from registration and evaluation; the information requirement for registration on substances of 1-10 tonnes is reduced; the role of the new EU chemical Agency was enhanced.

Basically, however, the REACH regulation will have a severe effect on competitiveness if no more corrections are made in the course of further legislative procedures. It will be a major challenge for the chemical industry as well as for its customers and downstream users to simultaneously survive in the global market and fulfil the conditions imposed by REACH with its heavy demands on resources.

Consequently, the European chemical industry is still faced with one of the major challenges of the last decades. In the years to come, the chemical industry will have to spend over 2 billion Euros for additional tests and the elaboration of probably more than 8000 registration dossiers. For many substances, profit margins are already now so small that those additional costs cannot be borne. CEFIC assumes that it will be no longer possible to produce 5-10\% of all substances on the European market. For downstream users this will mean the necessity to adapt formulations in the field of further processing, triggering extra costs of several billions of euros.

The fact that this is by no means an exaggeration is illustrated by experience gained in the review programmes for pesticides and biocides: in both areas, which are subject to the stronger regulation, i.e. to the authorisation procedure, more than half of all the active substances will no longer be on the market. Downstream users will thus face far-reaching rearrangements - in worst-case scenarios even having to go out of business - because they are no longer competitive on the global market due to the higher cost of their products. In the further processing sector, people will definitely be faced with additional costs amounting to several billions of euros.

Therefore, the entire industry must submit amendments in the remaining legislative process in order to improve the $\mathrm{REACH}$ regulation:

- Increased alignment of data requirements with the possible risk; stronger orientation of data requirements to potential risk (e.g. by easier waiving; i.e. no need for testing if there is no exposure).

- No duplication: no compulsory registration for previously regulated materials (e.g. when used in medical devices, plant protection products or biocides).

- Better definition of exposure categories to make communication along the supply chain more workable. Without a clear-cut definition of the newly introduced 'use and exposure categories', these cannot be applied in the daily practice of companies. The definition of these terms as decided by the EP should be adopted (EP, Article 3, nos. 12a, b);

- Protection of ownership rights to studies for 15 years (now 10 years).

- Fair sharing of data and costs between the companies in SIEF (Substance Information Exchange Forum) and in the consortia.

- Improved protection for confidential business information (mandatory data sharing);

- No time limit for authorisation if the risks are adequately controlled.

The Council of Ministers also decided that all new tests and analyses with the exception of physical-chemical parameters must, in future, be carried out according to the GLP rules (good laboratory practice) Under current law, only animal tests had to be carried out according to the GLP rules. For other investigations, only the usual demands on quality control and documentation were required. The additional GLP obligation would lead to a substantially higher cost, without improving the quality of results.

With the Common Position of the end of June 2006, we now have a version that must be compared with the proposals of the European Parliament. It must be said that it is not in the interest of the chemical industry to reopen the discussion about the entire package as this would jeopardize substantial compromises. However, the positions in important points are still far apart to the extent that the chemical industry supports the version of the Council of Ministers in the fields of 'Authorisation' and 'Duty of care' but supports that of the Parliament in the fields of 'Registration', 'Confidential- 
ity and access to information' as well as the 'Role of agent'.

\subsection{The Significance of the EU Market for the Swiss Chemical and Pharmaceutical Industry}

The chemical and pharmaceutical industry is Switzerland's second most important export branch. In 2005, companies exported a total volume of chemicals and pharmaceuticals to the value of CHF 54.7 billion. With around $60 \%$, a substantial part of chemical exports go to countries in the EU, in particular to Germany. More than $85 \%$ of all chemical imports come from the EU.

In all future discussions on the possible implementation of REACH in Switzerland, it must not be forgotten that - until 31st July 2005 - the Swiss chemical and pharmaceutical industry lived for decades with chemical legislation that was incipiently different from that of the EU and learned to live with the differences, whereby the EU market was always the most important one.

\section{The Challenges for the Swiss Chemical Industry}

\subsection{General Aspects}

The REACH challenges for companies in the chemical industry are manifold as well as different, dependent on the product ranges and export shares in detail - and may change to an enormous degree with REACH! One thing is clear: anyone supplying chemical products to the EU in future must comply with REACH. Given the obvious disadvantages of REACH at the moment, in particular, the enormous amount of administrative work (if not to say bureaucracy), the basic question also arises, however, as to whether we should introduce REACH to Switzerland at all. Months ago, an entrepreneur defined REACH as "the best piece of chemical legislation - for Chinese manufacturers", with whom our companies currently have to compete and probably even more so in the future.

\subsection{Exports to the EU}

It is already today clear that Swiss companies wishing to continue to export chemical products under REACH to the EU are faced with a significant additional package of costs. Each company must decide which substances or preparations it intends to leave or to introduce on European markets. For each product, a cost-benefit analysis must show whether it is worth fighting for competitive shares in the European market or whether it is economically more meaningful to orientate business to markets outside Europe in order to maintain or even gain market shares there with top-quality, but still affordable products.

\subsection{Switzerland as an Island in the Middle of a REACH Region}

Depending on the contents of article 6 (Notification of Substances in Imported Articles) of the draft REACH regulation on products, it may be advantageous for a downstream user to produce in Switzerland - or somewhere else outside the EU - and only deliver the finished articles or semifinished products to countries in the EU. Whether or not the application of REACH conditions on products, i.e. the textile dyestuff for a T-shirt manufactured and dyed in India would have to meet REACH criteria and stand up to WTO rules, is more than questionable. With the option 'not to implement REACH', companies in the Swiss chemical industry and downstream users would at any rate maintain a competitive advantage in the global market or even gain additional market opportunities. Whether this benefit justifies the disadvantage of a parallel existence of two chemical systems must be examined with care and from every perspective. This is probably the greatest challenge in connection with REACH since it might be decisive for the survival of individual companies in Switzerland.

\section{Conclusions}

REACH will bring about enormous changes to the future market for substances and preparations in the $\mathrm{EU}$; the changes will even affect global markets in some areas, particularly among users of substances and preparations. In Switzerland, different scenarios in terms of adapting to REACH are conceivable [5]: from its complete assumption through to being completely excluded. An in-depth analysis and discussion of the scenarios and their advantages and disadvantages will be necessary when the final version of the $\mathrm{REACH}$ regulation is available. This work has to be done by each company for their product portfolio and by SGCI Chemie Pharma Schweiz for the entire chemical and pharmaceutical industry. In this context, a REACH project of the Swiss authorities, especially the subproject 'Economic impacts of the implementation of REACH in Switzerland' would be of interest.

At any rate, the REACH proposal will not be automatically adopted in Switzerland. The fact that the Swiss legislative process will require time must not be considered as a disadvantage - we assume that a change in chemical law will be required. We might even learn something from the EU's possible mistakes.

Companies will do well to prepare themselves now for the coming situation and the consequences of REACH. There are major concerns and uncertainty: uncertainty regarding planning and the cost of implementation are top of the list. Companies in the chemical and pharmaceutical industry in Switzerland would be well advised to act now - without looking at the 'details' in the final REACH ordinance - to make an inventory of all their substances and preparations.

Received: August 14, 2006

[1] Council of the European Union, COM 2003, 0644 (03), http://europa.eu/eurlex/ en/com/pdf/2003/com2003_0644en.html.

[2] Council of the European Union, doc. $14228 / 05$ of 25.11 .2005 .

[3] Council of the European Union, doc. 15921/1/05 and doc. 15921/1/05 REV 1 of 09.03.2006 (consolidated version of agreed text).

[4] Council of the European Union, Common position adopted by the Council (REACH); (7524/06) Luxembourg, 2006, http://register.consilium.europa.eu/pdf/ en/06/st07/st07524.en06.pdf.

[5] Die Volkswirtschaft - Das Magazin für Wirtschaftspolitik 2005, (11), 54. 